\title{
Dietary choices of Indian immigrants living in the UK and the native population in India are negatively influenced by time constraints: A qualitative study
}

\author{
M. Sharma, J.C. Abayomi, N. Relph and P. Leadbetter \\ Faculty of Health, Social Care and Medicine, Edge Hill University, St Helens Road, Ormskirk, L39 4QP
}

Indian immigrants in the UK have a high prevalence of non-communicable diseases (NCD), mostly attributed to their diet and lifestyle choices and are often preventable. Acculturation induced dietary changes (transition from traditional to western diets) often adds to the deterioration of health in Indian immigrants. However, a similar dietary transition has been noticed in the country of origin (India), coupled with a significant rise in the onset of $\mathrm{NCD}^{(1)}$. This study aims to explore the dietary choices and the factors responsible for those choices, in Indian immigrants living in the UK, and compare it to the dietary choices of natives in India.

Participants were selected through purposive sampling, following previously identified independent variables such as age, religion, geographical region (state), socio-economic factors and gender in both the countries. Semi-structured, one-to-one interviews were conducted to collect detailed information about dietary choices and influencing factors from 18 Indian immigrants in the UK and 17 Indian natives in India. Interviews were audio-recorded and transcribed verbatim, then subjected to thematic analysis, guided by Braun \& Clark's step-by-step guide ${ }^{(2)}$.

Several preliminary themes have been identified, including 1) Time constraints, 2) Convenience, 3) Taste preferences, 4) Increased availability of ready to eat food and 5) Influence of globalised media. With respect to 'time constraints' - participants in both countries acknowledged that this has a great impact on their dietary choices; especially, in households where both the partners worked. They reported having very limited time to cook breakfast and/or lunch, for example

"Not much attention is paid on the afternoon meal, whatever is available basically" (Participant in the UK).

When asked about the reason, she replied "It is working time"

Similar responses were received from participants in India, reporting that traditional foods were only consumed on non-working days, with more time available: -

"She goes to her job everyday but like on some national holidays or on Sundays because she is at home, so particularly on those holidays, it's sometimes possible that er, she will cook some Indian delicacies" (Participant from India).

Increased use of takeaway food was reported on working days:

"on the days when we are not able to cook at home, then I do place orders and get the parcels from the nearby restaurants" (Participant from India).

These findings suggest that the changes in the dietary choices of Indian immigrants in the UK are not only due to acculturation. Time constraints, possibly due to women's roles changing from homemaker to bread-winner, with increased working outside the home in the UK and India, appears to be influencing the dietary choices of Indians living in both countries.

1. Pingali P (2007) Food Policy 32, 3, 281-298.

2. Braun V, Clarke V (2006). 3, 2, 77-101. 